\title{
Comparison of microbial communities from different Jinhua ham factories
}

\author{
Qingfeng $\mathrm{Ge}^{1,2}$, Yubin $\mathrm{Gu}^{2}$, Wangang Zhang ${ }^{1 *}$, Yongqi Yin' ${ }^{2}$, Hai Yu², Mangang Wu ${ }^{2}$, Zhijun Wang ${ }^{2}$ \\ and Guanghong Zhou ${ }^{1}$
}

\begin{abstract}
Microbes in different aged workshops play important roles in the flavor formation of Jinhua ham. However, microbial diversity, community structure and age related changes in workshops are poorly understood. The microbial community structure and diversity in Jinhua ham produced in factories that have 5, 15, and 30 years of history in processing hams were compared using the pyrosequencing technique. Results showed that 571,703 high-quality sequences were obtained and located in 242 genera belonging to 18 phyla. Bacterial diversity and microbial community structure were significantly different with the years of workshops. Three-phase model to characterize the changes of ham microbial communities was proposed. Gas chromatography-mass spectrometry assays indicated that the hams produced in different aged workshops have great differences in number and relative contents of volatiles compounds. These results suggest that different aged factories could form special and well-balanced microbial diversity, which may contribute to the unique flavor characteristics in Jinhua ham.
\end{abstract}

Keywords: Jinhua ham, Pyrosequencing, Microbial communities, Flavor, Aldehydes

\section{Introduction}

Previous studies have demonstrated that the unique and the broad diversity of flavors in ham are the result of complex reactions including lipid oxidation, Maillard reactions and protein degradation (Zhang et al. 2009; Zhou and Zhao 2007). These reactions mainly depend on enzymatic action of endogenous enzymes and microorganisms (Antequera et al. 1992; Petrova et al. 2015; Ventanas et al. 2008; Zhou and Zhao 2007). Hence, in the past two decades, the composition of microbial communities and main flora in ham has been widely investigated (Fulladosa et al. 2010; Martín et al. 2006). However, most of these researches have focused on the change of microbial diversity during the processing of ham. There are few reports concerning the differences in the microbial community structure in the ham that produced in different

\footnotetext{
*Correspondence: wangang.zhang@yahoo.com;

wangang.zhang@njau.edu.cn

${ }^{1}$ Key Lab of Meat Processing and Quality Control, Jiangsu Collaborative Innovation Center of Meat Production and Processing, Quality and Safety Control, College of Food Science and Technology, Nanjing Agricultural University, Nanjing 210095, Jiangsu, China

Full list of author information is available at the end of the article
}

manufacturing places. In the ham industry, it is generously accepted that the ham produced in different workshop has its unique flavor characteristics. The quality of ham is attributed to the maturing process of workshop which has a well-balanced microbial community structure and diversity in the ham. It is thus highly interesting to investigate the microorganism community structure of dry-cured ham produced in different workshops.

Community-level studies have become more precise with the application of culture-independent methods based on the direct detection of DNA in microbial ecosystems. The pyrosequencing techniques is the effective molecular tool for describing comprehensive diversities of microflora and has been successfully applied in fresh meat products (Xiao et al. 2013; Zhao et al. 2015) and sausages (Połka et al. 2015; Rebecchi et al. 2015) to understand the changes in the microbial populations during production or storage. Hence, applying the pyrosequencing technique in dry-cured ham can give new insight and comprehensive understanding of the microbial community structure.

Jinhua ham, a representative of traditional dry-cured meat product from Zhejiang Province in Eastern China, 
is considered as a high quality product with unique flavor. In the present study, the flavor characteristics of Jinhua ham produced in those workshops have 5,15 , and 30 years of history in processing hams and the structure and diversity of the microbial community of these hams were investigated by gas chromatography-mass spectrometry (GC-MS) and the $16 \mathrm{~S}$ rDNA gene pyrosequencing technique, respectively. The results were expected to provide insights into the microbial community structure and diversity among different aged workshops which produced special flavor Jinhua hams.

\section{Materials and methods}

\section{Processing of Jinhua ham and sampling}

Jinhua hams were processed in three workshops following the same traditional technology with same batch of green hams in Zhejiang Provincial Food Company, PR China. The traditional process divided into six phases: natural cooling, salting, soaking and washing, sun-drying, loft-aging and post-aging (Huan et al. 2005). According to the history of these three workshops, they were distinguished as JN (30 years), JD (15 years) and JM (5 years). All of these three workshops located in the Jindong district of Jinhua City, Zhejiang Province, their coordinates are $119^{\circ} 84^{\prime} \mathrm{E}-119^{\circ} 98^{\prime} \mathrm{E}$ and $29^{\circ} 28^{\prime} \mathrm{N}-29^{\circ} 46^{\prime} \mathrm{N}$.

At the middle of aging, hams were taken as samples for DNA extraction and volatile compounds analysis. Three hams in each workshop were randomly sampled, and three $3-\mathrm{cm}$-thick slices were taken from the central part of the Jinhua hams as described in Skrlep et al. were packed immediately and stored at $-40{ }^{\circ} \mathrm{C}$ for further analysis (Skrlep et al. 2012).

\section{Volatile compounds analysis}

Volatiles compounds analysis was carried out referring to the method of Lorenzo (Lorenzo 2014) and volatile compounds were extracted by solid phase micro-extraction technique (SPME) with a $10 \mathrm{~mm}$ long and $75 \mu \mathrm{m}$ thick fiber coated with poly-dime-thylsiloxane. Twenty grams of minced sausages were used to extract volatiles compounds. Before the collection of volatiles, the fiber was preconditioned in the GC injection port at $250{ }^{\circ} \mathrm{C}$ for $20 \mathrm{~min}$ and then inserted into conical flask through the septum, afterwards exposed to the headspace for $40 \mathrm{~min}$ at $60{ }^{\circ} \mathrm{C}$ in a water bath. GC-MS analysis was performed with a GC-MS apparatus (Thermo Fisher Scientific, MA, USA). DB-5MS capillary column $(30 \mathrm{~m} \times 0.25 \mathrm{~mm} \times 0.25 \mu \mathrm{m}$, J\&W Scientific, Palo Alto, CA, USA) was used for the separation, the carrier gas was helium with the flow rate of $180 \mathrm{~mL} / \mathrm{min}$ and the SPME fiber would be maintained at $250^{\circ} \mathrm{C}$. The temperature program was: from initial temperature $40{ }^{\circ} \mathrm{C}(1 \mathrm{~min}$ hold) to $130{ }^{\circ} \mathrm{C}$ at $5{ }^{\circ} \mathrm{C} / \mathrm{min}$, to $200{ }^{\circ} \mathrm{C}$ at $8{ }^{\circ} \mathrm{C} / \mathrm{min}$, to
$250{ }^{\circ} \mathrm{C}$ at $12{ }^{\circ} \mathrm{C} / \mathrm{min}$ and held for $7 \mathrm{~min}$ at $250{ }^{\circ} \mathrm{C}$. The EI of mass spectrometer and GC-MS transfer line were all operated at $250{ }^{\circ} \mathrm{C}$, detector voltage was $350 \mathrm{~V}$, emission current was $150 \mu \mathrm{A}$, rate was $1 \mathrm{scan} / \mathrm{s}$ and $m / z$ range was 33-500 for data collection. Compared with spectra from the NIST, the volatile compounds were identified and then quantified by calculating the ratio of individual compound peak area with the total peak area relatively.

\section{Pyrosequencing for 165 rDNA}

According to the manufacturer's instruction, the microbial DNA was extracted from the hams with PowerFood Microbial DNA Isolation kit (MO BIO Laboratories, Inc., USA). The V3 hypervariable region of the $16 \mathrm{~S}$ rDNA was PCR amplified from the microbial genomic DNA using universal primer (forward primers: $5^{\prime}$-ACTCCTACGGGAGGCAGCAG-3', reverse primers: 5'-TTACCGCGGCTGCTGGCAC-3'). PCR was subjected to 1 cycle of $98{ }^{\circ} \mathrm{C}$ for $5 \mathrm{~min}$, followed by 25 cycles of denaturation at $98{ }^{\circ} \mathrm{C}$ for $30 \mathrm{~s}$, annealing at $58{ }^{\circ} \mathrm{C}$ for $30 \mathrm{~s}$ and extension at $72{ }^{\circ} \mathrm{C}$ for $30 \mathrm{~s}$, and finally extension at $72{ }^{\circ} \mathrm{C}$ for $5 \mathrm{~min}$. Barcoded V3 amplicon was sequenced by Illumina Miseq at Personal Biotechnology Co., Ltd (Shanghai, China) using the pair-end method. All related sequence data have been deposited in the National Center for Biotechnology Information (NCBI) as a bio-project (BioProject ID: PRJNA354505, Accession Number: SRP093702). The samples were given the accessions numbers as SAMN 06046958 -SAMN06046966.

\section{Pyrosequencing data analysis}

After pyrosequencing, all readings were screened and filtered using QIIME 1.6.0 software (Caporaso et al. 2010). Sequences reads with an average quality score lower than 25 , ambiguous bases, homopolymer $>7$ bases, containing primer mismatches, or reads length shorter than $150 \mathrm{bp}$ were removed. For V3 pair-end read, only sequences that overlapped more than $10 \mathrm{bp}$ and without any mismatches were assembled. Operational taxonomic units (OTUs) were picked only if they had similar values of $97 \%$ or higher. Rarefaction curves and Venn plot were generated (Kõljalg et al. 2013). Alpha diversity was evaluated by community richness (Chao1 and ACE) (Pitta et al. 2010) and community diversity (Shannon and Simpson species) (Mahaffee and Kloepper 1997; Shannon 2001). All described analyses were performed using version 1.32.1 of MOTHUR software package (Schloss et al. 2009). Subsequent taxonomic affiliations were then obtained using the RDP classifier (http://rdp.cme.msu.edu/) with a confidence threshold cutoff of 0.8 to determine the taxonomy of the sequences (Wang et al. 2007). The abundances (percentages) were compared at the genus level $(0.05$ OTU) in each sample. The heat map were described using 
the statistical software package $\mathrm{R}$ (Ihaka and Gentleman 1996). The functional composition of communities were described using the statistical software PICRUSt (Langille et al. 2013) and annotated to their biological function according to KEGG (http://www.kegg.jp/kegg/pathway. html).

\section{Statistical analyses}

All experiments in this study were repeated at least three times in independent experiments. Means and standard deviations were computed according to the experimental data. One way analysis of variance (ANOVA) with Tukey's test was conducted on the data, and a $P$ value at 0.05 was considered significant.

\section{Results}

\section{Volatile flavor compounds in Jinhua ham}

To investigate the influences of long-term batch fermenting on the production of volatile flavor compounds, the volatile compositions in the Jinhua ham produced from the different aged workshops were detected using the GC-MS system (Fig. 1). Difference of total number of volatiles compounds and their relative contents among JN (30 years), JD (15 years) and JM (5 years) samples were observed. In this study, 72 volatile compounds that clustered in 8 chemical families were identified and quantified. Results indicated that the most abundant chemical family in flavor of Jinhua ham was aldehydes. After postaging, the aldehydes contents in $\mathrm{JN}$ reached to $49.8 \%$ with 1.36- and 1.50-fold of JM and JD, respectively (Fig. 1). Similarly, based on relative peak area, ketones were the more abundant in the ham produced in JN, whereas acids, esters, ethers and hydrocarbons were more abundant in JM and JD. The results show that Jinhua hams have great differences in flavor among different workshop.

\section{Amplification and sequencing of Jinhua ham bacteria $16 \mathrm{~S}$ rDNA gene sequences}

The libraries containing the $16 \mathrm{~S}$ rDNA gene sequences of bacteria targeting the $\mathrm{V} 3$ hypervariable region fragments of $16 \mathrm{~S}$ rDNA gene were constructed. The entire pyrosequencing data set from the three samples contained 614,495 sequences. After filtering, 571,703 high-quality sequences (93.04\% of the total sequences) remained with an average read length of $159 \mathrm{bp}$.

It is interesting that Chao1 and ACE in JN were higher than that in JD while lower than in JM (Table 1). Meanwhile, to estimate the overall diversity of ham bacteria, the Shannon and Simpson species richness index in each sample was also calculated (Table 1). The results showed that the ham bacteria were obviously different among the $\mathrm{JN}, \mathrm{JM}$ and JD, suggesting that different years fermenting workshops had their own balanced microbial community structure and diversity.

\section{Microbiota composition in ham markedly diversified in aged workshop}

The Venn plot indicated that 604 OTUs were common across all ham corresponding to some families of bacteria existing among three hams (Fig. 2). The microbiota in Jinhua ham was constituted of nineteen phyla and the vast majority of sequences belong to one of the four major phylas: Bacteroidetes, Actinobacteria, Proteobacteria or Firmicutes (Fig. 3a). The abundance of the remaining phyla was less than $1.5 \%$ of total sequences including Acidobacteria, Chloroflexi, Cyanobacteria, Fibrobacteres, Fusobacteria, Lentisphaerae, OP8, OP9, Spirochaetes, Synergistetes, TM7, Tenericutes, WPS-2 and Thermi. Among all hams, Firmicutes was the most predominant microbiota and its abundance was more than $52.00 \%$ even as high as $81.80 \%$ in JN. However, the abundance of Bacteroidetes and Proteobacteria in JN both lower than that in JD and JM. Additionally, The total bacterial sequences from the three ham samples located in 242 genera. These bacterial genera and their abundances in the JD, JN and JM ham were shown in the heat map (Fig. 4). The map presents that the bacteria in ham were markedly different in composition form. The clustering analysis led to the division of the 242 genera into 6 prominent categories (Fig. 4). The intensity of genera belonging to clusters I in JD and JN was higher than that in JM. Those genera in clusters II abounded in JN ham and their abundance in JD and JM was relatively lower. It was noted that the abundance of genera belonging to clusters III, IV and V was higher in JM, but they showed significant diversity in JD and JN. Differences among the genera in clusters VI also presented in three ham samples. To observe the changes in the ham closely, the 11 most abundant genera that appeared in all three samples was compared. The results showed that after a decade of fermentation acclimation, the distribution differences of bacteria were reduced (Fig. 3b). For example, among all hams, the most frequently detected genus was Staphylococcus which changed in different ham samples. This genus accounted for $79.71 \%$ of the total in JN, but decreased markedly in JD (48.58\%) and JM (48.16\%). Actinosynnemataceae sensu stricto ranked the second only to Staphylococcus in JD, but the genus was less in JM. It is also noted that there were many bacterial sequences whose taxonomic status could not be defined. Furthermore, some sequences could not be defined of their taxonomic status, even at the phylum level. The changes of these unclassified bacteria became greatly among different hams and its composition markedly increased in JM. 

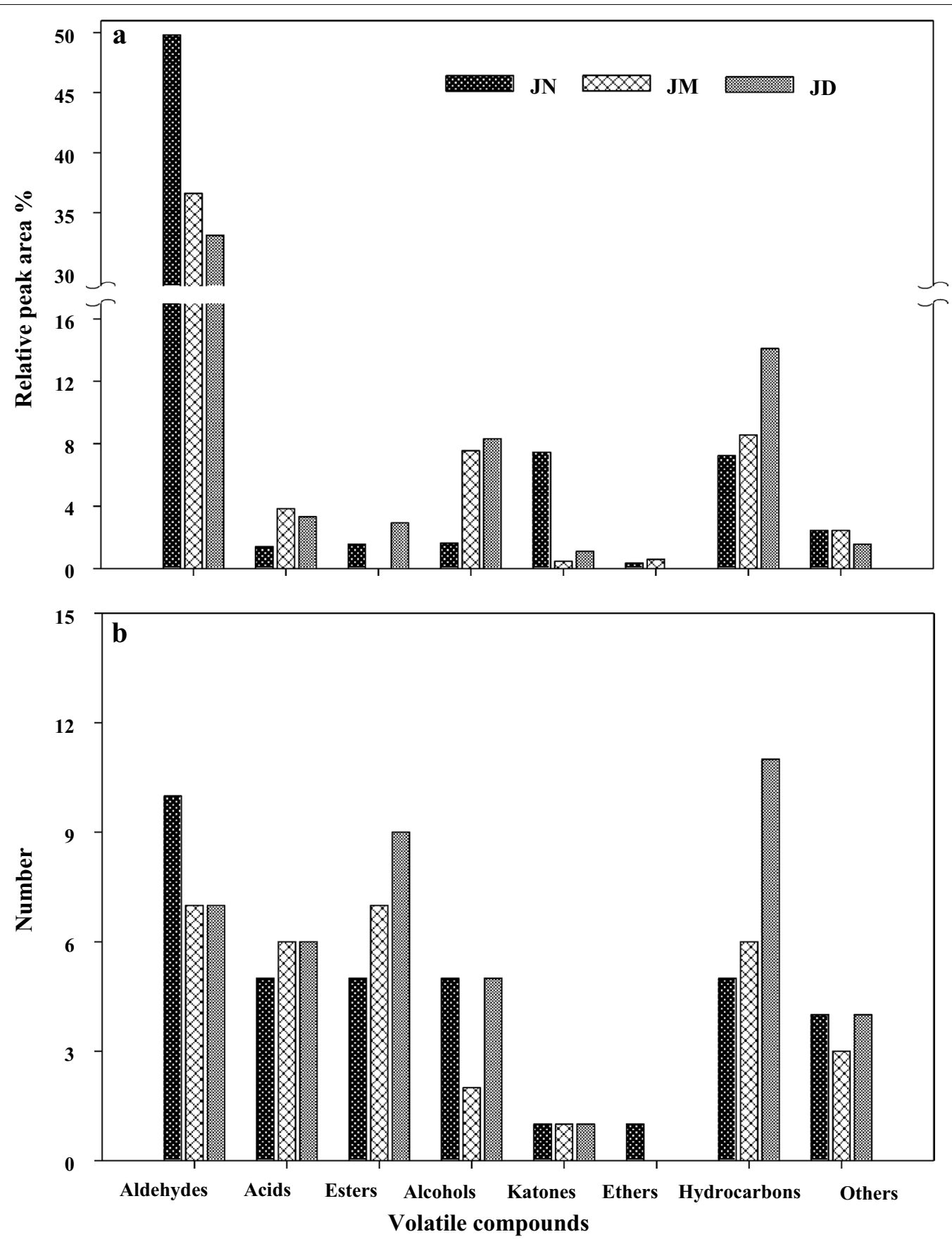

Fig. 1 Volatile flavor compounds detected in Jinhua ham after post-ripening. a The relative peak area of volatile flavor compounds. $\mathbf{b}$ The number of volatile flavor compounds

Table 1 Alpha diversity in Jinhua ham

\begin{tabular}{|c|c|c|c|c|c|c|}
\hline \multirow[t]{2}{*}{ Sample } & \multirow[t]{2}{*}{ Reads } & \multirow[t]{2}{*}{ High-quality sequences } & \multicolumn{2}{|c|}{ Abundance index } & \multicolumn{2}{|c|}{ Diversity index } \\
\hline & & & Chao1 & ACE & Shannon & Simpson \\
\hline JN & 83,687 & 80,935 & 597.5 & 598.4 & 0.4491 & 4.5668 \\
\hline$J M$ & 84,282 & 80,776 & 827.8 & 844.4 & 0.7735 & 4.9827 \\
\hline JD & 62,201 & 54,800 & 547.5 & 533.9 & 0.6512 & 3.2359 \\
\hline
\end{tabular}




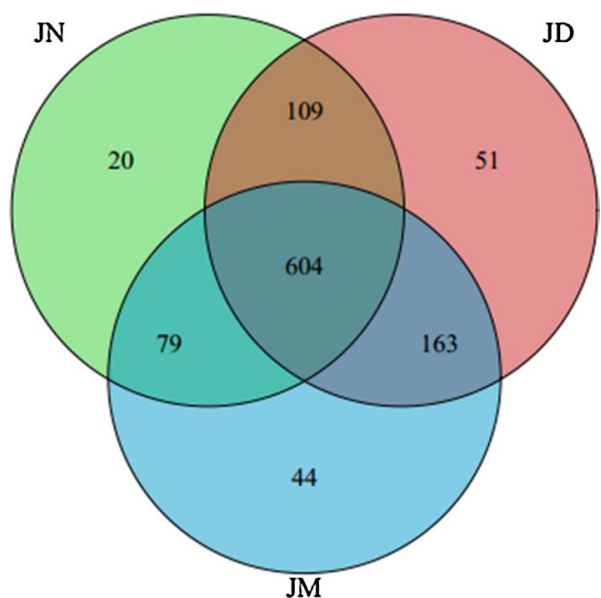

Fig. 2 Venn plot for microbial diversity among ham produced from different workshop. The figures in different compartments mean the numbers of sequences specific for or common to ham workshop
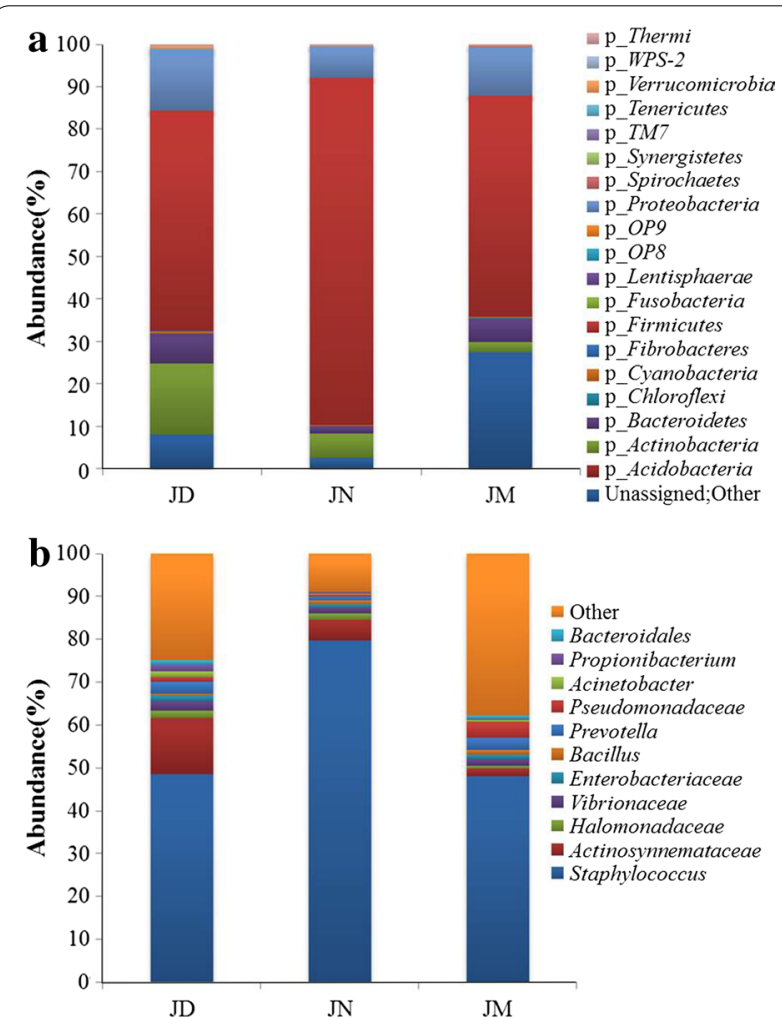

Fig. 3 Changes in abundance of bacterial phyla based on 16S rDNA sequencing. a phyla-based; $\mathbf{b}$ family-based. In the legend, " $k$ " stands for kingdom and " $p$ " for phyla

\section{Functional composition diversity varying with age of workshops}

Predicted functional class analysis showed that these OTUs were divided into 7 functional classes including

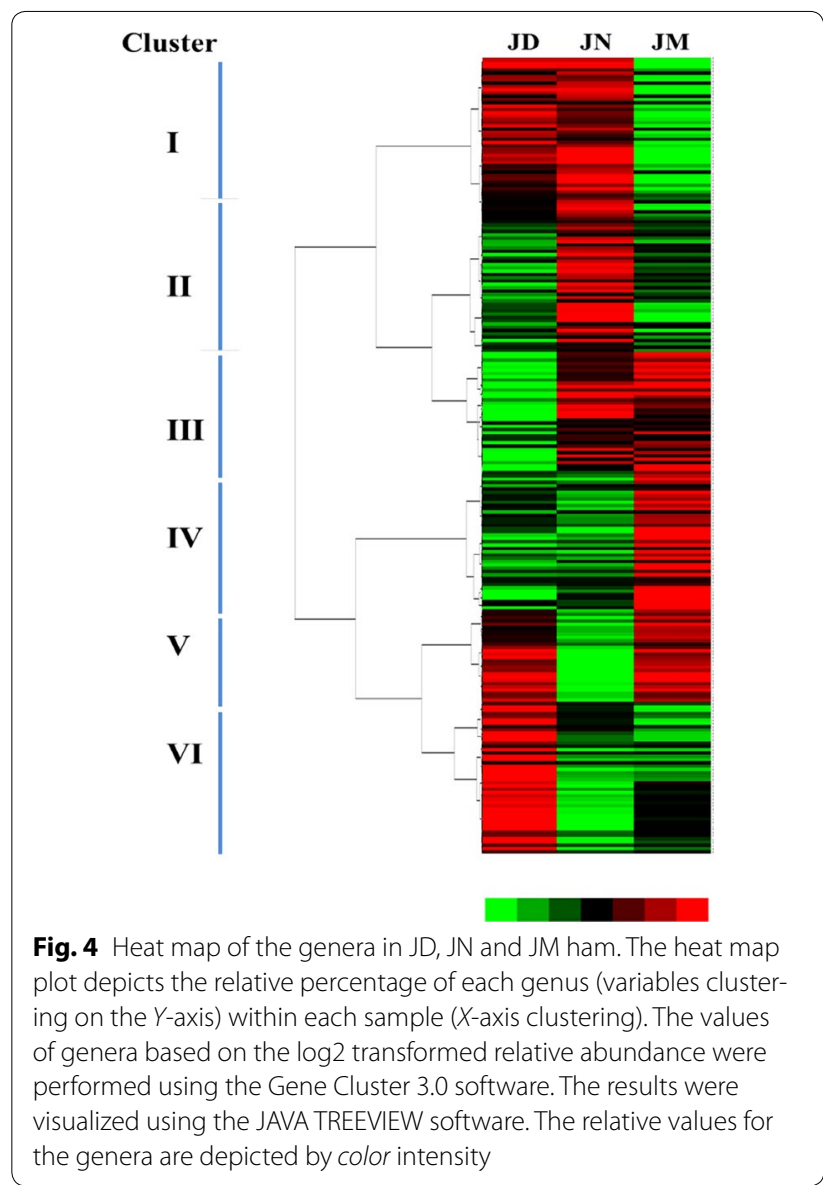

cellular processes, environmental information processing, genetic information processing, human diseases, metabolism, organismal system and unclassified. In the present study, in order to effectively analyze the difference in the ham, OTUs involved in metabolism that appeared in Jinhua hams was compared and it showed their own different metabolic process (Fig. 5). The abundance of amino acid metabolism-related and carbohydrate metabolism-related OTUs were significantly higher than other metabolism-related. Specifically, the entire metabolism-related OTUs in different hams had sharp distinction indicating that the different years fermenting benefits the Jinhua ham flavor formation for their difference of complex metabolism, which depends on their own well-balanced bacteria community.

\section{Discussion}

The workshop microbiota is recognized to play a role in the flavors of Jinhua ham. In the present study, microbial community structure and diversity of different ages of ham workshops were investigated by the high-throughput pyrosequencing technique. Our results provide comprehensive understanding of microbial community 


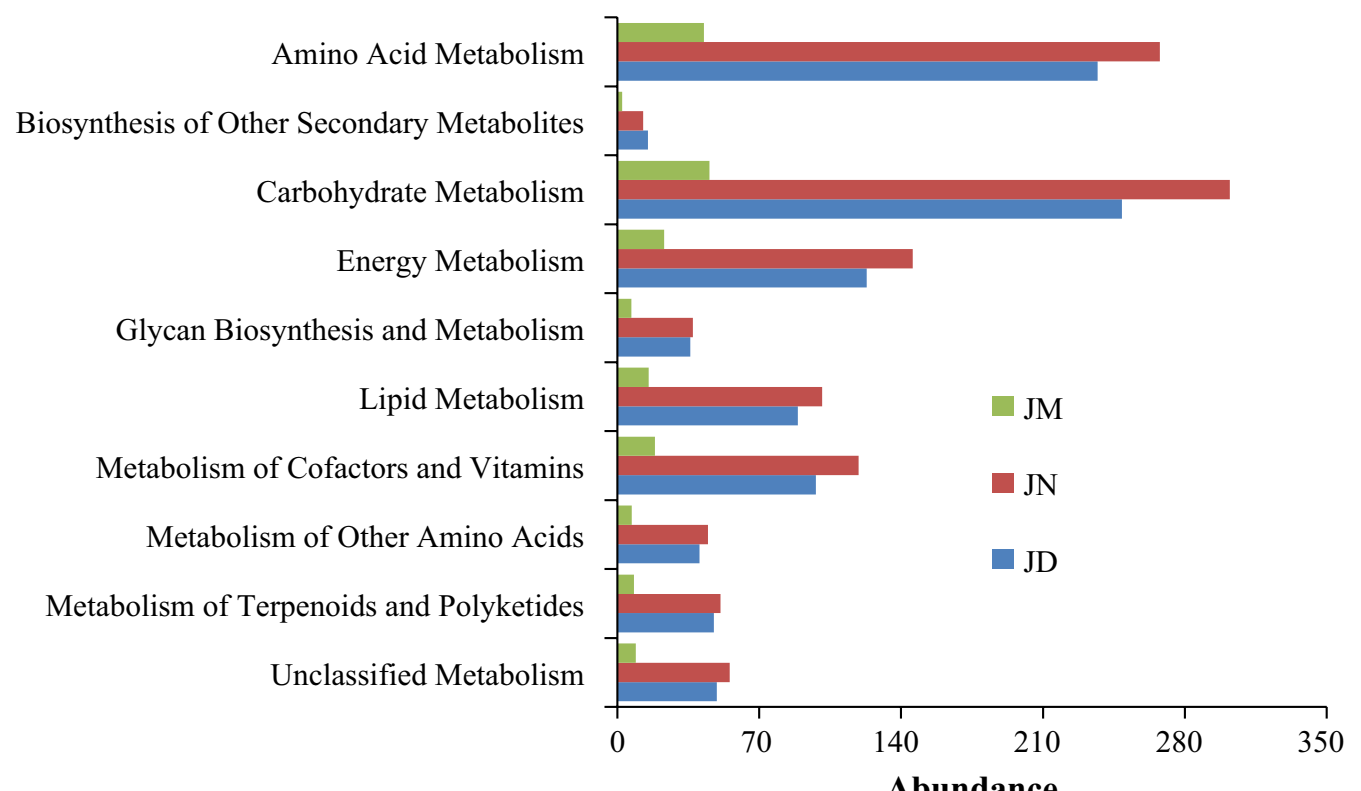

Fig. 5 Functional genes related to metabolism in Jinhua ham. The functions of OTUs were assigned according to KEGG

structure and diversity among different aged workshops which produced special flavor Jinhua hams.

\section{Difference in Workshop lead to entirely different sets of microbial community structure}

Microorganisms in ham are involved in the fermentation process to produce the aromatics which determine the ham flavor style which were routinely selected by the fermentation process. The genera Staphylococcus (Cordero and Zumalacarregui 2000; Fulladosa et al. 2010) were identified from dry-cured ham samples by cultured or un-cultured methods in previous studies. Here, we identified 242 genera as the core microbiota in Jinhua ham samples using the pyrosequencing technique. Consistent with previous reports, despite the shifted percentage in different ham, the most frequently detected genus also was Staphylococcus among all hams in the present study. Additionally, the present study indicated that microbial communities in the JM (5-year workshop) were different from those in the JD (15-year) and JN (30-year). The diversity of prokaryotes decreased with workshop age and sustained advantage and balance in the JN (Fig. 4), while those in the JD were in the transition state. According to our results, three distinct phases were separated by the changes of workshop microbial communities. Phase A was high in diversity and species richness which was possibly resulted from biogeochemical environment similar to the surrounding air environment. At this phase, the abundance of genera belonging to clusters III, IV and $\mathrm{V}$ was higher (Fig. 4). Additionally, there were so many unassigned bacteria in phase A but decreased in phase B (Fig. 3). The community structure of Phase B dramatically changed and significant decreased in prokaryotic diversity. This could be due to the fact that the microbial community was optimized and adapted at very different environmental conditions (e.g., temperature and humidity) created by the Jinhua ham process in the workshop. However, the abundance of Actinobacteria and Proteobacteria increased (Fig. 3). The abundance of genera belonging to clusters $\mathrm{I}$ in Phase $\mathrm{B}$ was consistent with Phase $C$. Phase $C$ was the relative mature period of the ham microbial community. Microbial diversity was stable in this phase and was significantly lower than in the young workshop. The specific microbial distribution in the ham may be resulted in periodic fermentation and enrichment for more than 30 years without interruption. Moreover, mutual collaborations and interactions among different bacteria species could lead to a well-balanced bacteria community in the biogeochemical environment resulting in ham flavor different.

\section{Dominant bacteria communities and their relationships to Jinhua ham flavors}

Bacteria communities play a crucial role in the production of flavor of the dry-cured meat product (Fadda et al. 2010; Kaban 2013). Volatile compounds are generated from the catabolism of proteins, lipids, and carbohydrates through the action of microbial and endogenous enzymes during process due to a high natural microbiota background (Lücke 1996; Zeuthen 2007). The total number of 
volatile compounds identified in the present study and/or their relative contents were different. Studies have shown the dominant volatile compounds in Bayonne, Jinhua, Corsican, Iberian, Parma and Serrano dry-cured hams were aldehydes (Huan et al. 2005; Mottram 1998). It is not surprising that the content of aldehydes increased over age of the workshop which had well-balanced bacteria community in the present study. Moreover, hexanal with an odor of green leaves is the dominated profile of aldehydes and is synthesized during the oxidation of unsaturated fatty acids. Hexanal was found at the highest level in JN while lowest in JM. Previous works have been carried out to study the changes in volatile aldehydes and ketones in dry-cured ham. Those results showed that flavor formation by secondary metabolism of microorganisms, especially amino acid catabolism in which methyl-branched aldehydes and methyl ketones were generated (Hinrichsen and Andersen 1994; NarváezRivas et al. 2014). Additionally, since OTUs involved in metabolism of terpenoids and polyketides, amino acids, lipid and carbohydrate in JN differed from in JM and its flavor could have their own typical characteristics. In conclusions, the microbial diversity and community structure markedly changed in different aged workshops which corresponded to the strong flavoring characteristics of Jinhua ham.

In conclusion, GC-MS assays indicated that remarkably difference among Jinhua hams concerning number of volatiles compounds and their relative contents. Hams produced in the older workshop contained the higher concentrations of aldehydes that are important for the ham flavor. Meanwhile, bacterial diversity and microbial community structure were significantly different among three factories. Three-phase model to characterize the changes of ham microbial communities was demonstrated. Importantly, these results indicated that aged factories could accumulate adaptive microbes, and wellbalanced microbial diversity was responsible for the production of more flavorful Jinhua ham.

\section{Abbreviations}

DNA: deoxyribonucleic acid; GC-MS: gas chromatography-mass spectrometry; SPME: solid phase micro-extraction technique; OTUs: operational taxonomic units; NIST: National Institute of Standards and Technology; PCR: polymerase chain reaction; NCBI: National Center for Biotechnology Information.
\end{abstract}

\section{Authors' contributions \\ $G Q, G Y$ and $Y Y$ conducted the experiments and wrote the manuscript; $Y H$ and WM performed the data analyses; ZW supervised the research and revised the manuscript; WZ and ZG supervised the research; All authors read and approved the final manuscript.}

\section{Author details}

${ }^{1}$ Key Lab of Meat Processing and Quality Control, Jiangsu Collaborative Innovation Center of Meat Production and Processing, Quality and Safety Control, College of Food Science and Technology, Nanjing Agricultural University,
Nanjing 210095, Jiangsu, China. ${ }^{2}$ College of Food Science and Engineering, Yangzhou University, Yangzhou 225127, Jiangsu, China.

\section{Acknowledgements}

We thank associate professor Runqiang Yang (College of Food Science and Technology, Nanjing Agricultural University) for his insightful suggestions and helpful discussions.

\section{Competing interests}

The authors declare that they have no competing interests.

\section{Availability of data and materials}

All related sequence data obtained in this study have been deposited in the NCBI as a bio-project (BioProject ID: PRJNA354505, Accession number: SRP093702)

\section{Ethical approval}

This article does not contain any studies with human participants or animals performed by any of the authors.

\section{Funding}

The word was supported by the Sci-Technology program of Jiangsu Province (BE2014328, BE2014359, BN2014164,BN2014005) and the New Century Talent Project of Yangzhou University.

Received: 25 January 2017 Accepted: 30 January 2017

Published online: 13 February 2017

\section{References}

Antequera T, López-Bote C, Córdoba J, García C, Asensio M, Ventanas J, García-Regueiro J, Díaz I (1992) Lipid oxidative changes in the processing of Iberian pig hams. Food Chem 45(2):105-110. doi:10.1016/0308-8146(92)90018-W

Caporaso JG, Kuczynski J, Stombaugh J, Bittinger K, Bushman FD, Costello EK, Fierer N, Pena AG, Goodrich JK, Gordon JI (2010) QIIME allows analysis of high-throughput community sequencing data. Nat Methods 7(5):335336. doi:10.1038/nmeth.f.303

Cordero MR, Zumalacarregui JM (2000) Characterization of Micrococcaceae isolated from salt used for Spanish dry-cured ham. Lett Appl Microbiol 31 (4):303-306. doi:10.1046/j.1472-765x.2000.00818.x

Fadda S, Lopez C, Vignolo G (2010) Role of lactic acid bacteria during meat conditioning and fermentation: peptides generated as sensorial and hygienic biomarkers. Meat Sci 86(1):66-79. doi:10.1016/j. meatsci.2010.04.023

Fulladosa E, Garriga M, Martín B, Guàrdia M, García-Regueiro J, Arnau J (2010) Volatile profile and microbiological characterization of hollow defect in dry-cured ham. Meat Sci 86(3):801-807. doi:10.1016/j.meatsci.2010.06.025

Hinrichsen LL, Andersen HJ (1994) Volatile compounds and chemical changes in cured pork: role of three halotolerant bacteria. J Agr Food Chem 42(7):1537-1542. doi:10.1021/jf00043a027

Huan YJ, Zhou GH, Zhao GM, Xu XL, Peng ZQ (2005) Changes in flavor compounds of dry-cured Chinese Jinhua ham during processing. Meat Sci 71(2):291-299. doi:10.1016/j.meatsci.2005.03.025

Ihaka R, Gentleman R (1996) R: a language for data analysis and graphics. J Comput Graph Stat 5(3):299-314

Kaban G (2013) Sucuk and pastirma: microbiological changes and formation of volatile compounds. Meat Sci 95(4):912-918. doi:10.1016/j. meatsci.2013.03.021

Köljalg U, Nilsson RH, Abarenkov K, Tedersoo L, Taylor AF, Bahram M, Bates ST, Bruns TD, Bengtsson-Palme J, Callaghan TM (2013) Towards a unified paradigm for sequence-based identification of fungi. Mol Ecol 22(21):5271-5277. doi:10.1111/mec.12481

Langille MG, Zaneveld J, Caporaso JG, McDonald D, Knights D, Reyes JA, Clemente JC, Burkepile DE, Thurber RLV, Knight R (2013) Predictive functional profiling of microbial communities using 165 rRNA marker gene sequences. Nat Biotechnol 31(9):814-821. doi:10.1038/nbt.2676 
Lorenzo JM (2014) Changes on physico-chemical, textural, lipolysis and volatile compounds during the manufacture of dry-cured foal "cecina". Meat Sci 96(1):256-263. doi:10.1016/j.meatsci.2013.06.026

Lücke FK (1996) Lactic acid bacteria involved in food fermentations and their present and future uses in food industry lactic acid bacteria. Springer, Berlin, pp 81-99

Mahaffee W, Kloepper J (1997) Temporal changes in the bacterial communities of soil, rhizosphere, and endorhiza associated with field-grown cucumber (Cucumis sativus L.). Microb Ecol 34(3):210-223. doi:10.1007/ s002489900050

Martín A, Córdoba JJ, Aranda E, Córdoba MG, Asensio MA (2006) Contribution of a selected fungal population to the volatile compounds on dry-cured ham. Inter J Food Microb 110(1):8-18. doi:10.1016/j. ijfoodmicro.2006.01.031

Mottram DS (1998) Flavour formation in meat and meat products: a review. Food Chem 62(4):415-424. doi:10.1016/S0308-8146(98)00076-4

Narváez-Rivas M, Gallardo E, León-Camacho M (2014) Chemical changes in volatile aldehydes and ketones from subcutaneous fat during ripening of Iberian dry-cured ham. Prediction of the curing time. Food Res Inter 55(2):381-390. doi:10.1016/j.foodres.2013.11.029

Petrova I, Aasen IM, Rustad T, Eikevik TM (2015) Manufacture of dry-cured ham: a review. Part 1. Biochemical changes during the technological process. Eur Food Res Technol 241(5):587-599. doi:10.1007/s00217-015-2490-2

Pitta DW, Pinchak WE, Dowd SE, Osterstock J, Gontcharova V, Youn E, Dorton K, Yoon I, Min BR, Fulford J (2010) Rumen bacterial diversity dynamics associated with changing from bermudagrass hay to grazed winter wheat diets. Microb Ecol 59(3):511-522. doi:10.1007/s00248-009-9609-6

Połka J, Rebecchi A, Pisacane V, Morelli L, Puglisi E (2015) Bacterial diversity in typical Italian salami at different ripening stages as revealed by highthroughput sequencing of $16 \mathrm{~S}$ rRNA amplicons. Food Microb 46:342356. doi:10.1016/j.fm.2014.08.023

Rebecchi A, Pisacane V, Miragoli F, Polka J, Falasconi I, Morelli L, Puglisi E (2015) High-throughput assessment of bacterial ecology in hog, cow and ovine casings used in sausages production. Inter J Food Microb 212:49-59. doi:10.1016/j.ijfoodmicro.2015.04.047
Schloss PD, Westcott SL, Ryabin T, Hall JR, Hartmann M, Hollister EB, Lesniewski RA, Oakley BB, Parks DH, Robinson CJ (2009) Introducing mothur: opensource, platform-independent, community-supported software for describing and comparing microbial communities. Appl Environ Microb 75(23):7537-7541. doi:10.1128/AEM.01541-09

Shannon CE (2001) A mathematical theory of communication. Mobile Comput Commun R 5(1):3-55

Skrlep M, Candek-Potokar M, Zlender B, Robert N, Sante-Lhoutellier V, Gou P (2012) PRKAG3 and CAST genetic polymorphisms and quality traits of dry-cured hams-III. Associations in Slovenian dry-cured ham Kraski prsut and their dependence on processing. Meat Sci 92(4):360-365. doi:10.1016/j.meatsci.2012.06.021

Ventanas S, Estevez M, Andrés Al, Ruiz J (2008) Analysis of volatile compounds of Iberian dry-cured loins with different intramuscular fat contents using SPME-DED. Meat Sci 79(1):172-180. doi:10.1016/j.meatsci.2007.08.011

Wang Q, Garrity GM, Tiedje JM, Cole JR (2007) Naive Bayesian classifier for rapid assignment of rRNA sequences into the new bacterial taxonomy. Appl Environ Microb 73(16):5261-5267. doi:10.1128/AEM.00062-07

Xiao X, Dong Y, Zhu Y, Cui H (2013) Bacterial diversity analysis of Zhenjiang Yao meat during refrigerated and vacuum-packed storage by 454 pyrosequencing. Curr Microb 66(4):398-405. doi:10.1007/s00284-012-0286-1

Zeuthen P (2007) A historical perspective of meat fermentation. In: Toldra F (ed) Meat fermentation worldwide: history and principles. Handbook of fermented meat and poultry, vol 1. Blackwell, USA, pp 3-8

Zhang JH, Zhen Z, Zhang WG, Zeng T, Zhou G (2009) Effect of intensifying high-temperature ripening on proteolysis, lipolysis and flavor of Jinhua ham. J Sci Food Agric 89(5):834-842. doi:10.1002/jsfa.3521

Zhao F, Zhou G, Ye K, Wang S, Xu X, Li C (2015) Microbial changes in vacuumpacked chilled pork during storage. Meat Sci 100(100):145-149. doi:10.1016/j.meatsci.2014.10.004

Zhou G, Zhao G (2007) Biochemical changes during processing of traditional Jinhua ham. Meat Sci 77(1):114-120. doi:10.1016/j.meatsci.2007.03.028

\section{Submit your manuscript to a SpringerOpen ${ }^{\circ}$ journal and benefit from:}

- Convenient online submission

- Rigorous peer review

- Immediate publication on acceptance

- Open access: articles freely available online

- High visibility within the field

- Retaining the copyright to your article

Submit your next manuscript at springeropen.com 